\title{
Dalla pop art alla pittura antica
}

Visioni, limiti e discontinuo negli scritti sull'arte di Celati

\section{Maria Teresa De Palma}

\section{OpenEdition}

\section{Journals}

Edizione digitale

URL: https://journals.openedition.org/cher/656

DOI: $10.4000 /$ cher.656

ISSN: 2803-5992

\section{Editore}

Presses universitaires de Strasbourg

\section{Edizione cartacea}

Data di pubblicazione: 9 juillet 2020

Paginazione: 19-32

ISBN: 979-10-344-0068-3

ISSN: 1968-035X

Notizia bibliografica digitale

Maria Teresa De Palma, «Dalla pop art alla pittura antica», reCHERches [Online], 24 | 2020, online dal 20 septembre 2021, consultato il 19 novembre 2021. URL: http://journals.openedition.org/cher/656 ; DOI: https://doi.org/10.4000/cher.656 


\title{
Dalla pop art alla pittura antica \\ Visioni, limiti e discontinuo negli scritti sull'arte di Celati
}

\author{
Maria Teresa De Palma
}

\begin{abstract}
T 'immagine, il paragone pittorico, la meditazione sul visivo sono in Celati _altrettante «favole identitarie» ${ }^{1}$ : mezzi cioè per precisare e riflettere le proprie idee sulla scrittura, intesa a un tempo come forma dell'espressione e modalità vitale. D'altronde, è soprattutto nella pratica del commento - più che in quella dello scritto sistematico - che nel tempo si va precisando in Celati una riflessione, coerente seppur discontinua, sullo statuto dell'immagine, nutrita al fondo da precoci letture fenomenologiche e certamente acuita dalla frequentazione, a partire dagli anni Ottanta, del fotografo Luigi Ghirri.
\end{abstract}

I brani di Celati sulla pittura, in particolare, che qui saranno oggetto d'analisi, non si rivelano spesso altro che tracce, abbozzi, linee d'indagine sviluppate a partire da una ricerca parallela attorno all'uso e alla natura del letterario; tracce che, tuttavia, se lette nel loro insieme, rivelano una sostanziale continuità di fondo e, quel che più interessa, una concordanza stupefacente rispetto al più generale "pensiero estetico" dello scrittore. Perché fin da adesso, quasi a premessa di tutto, è collocabile un primo, fondamentale punto fermo: in Celati non si darà mai scollamento o distanziazione tra immagine e parola, ma invece una sostanziale complicità, un'adesione evidentissima. Anticipando un poco, si citeranno qui le parole di un saggio su cui torneremo, lo scritto su «Beckett e le sorti dell'espressione», dove si parla apertamente dei «nostri artefatti di parole o di immagini», dando cioè per assunto che non esista separazione, ma una netta continuità tra codici e linguaggi. E questo perché, dai tempi del noviziato fino ai più recenti scritti, "tutto ciò che spunta dalla penna o dal pennello» (Celati 1/18: $43^{2}$ ) è per Celati accomunato da identiche modalità, intenzioni e inclinazioni artigiane.

1 Prendo qui a prestito una definizione di Andrea Cortellessa, in quel caso riferita alla traduzione celatiana del Bartleby di Melville, cfr. Cortellessa 2005: 551.

2 Le citazioni tratte dai materiali inediti conservati presso l'Archivio Celati a Reggio Emilia recano, accanto al nome dell'autore, il numero della busta entro cui è conservato il documento. In bibliografia, i titoli non autoriali di tali materiali 
A chi voglia approfondire il rapporto tra Celati e le arti visive, dunque, si offre oramai un corpus vastissimo di scritti, interventi critici, notarelle diaristiche a taglio più o meno personale. Prima di inoltrarci nella lettura di questi brani sparsi - ché quest'intervento conserverà comunque l'andamento discontinuo del collage, e la passione malinconica della citazione -, sarà bene anticipare alcuni dei nodi concettuali attorno a cui andrà organizzandosi l'analisi; e sarà bene chiedersi fin da ora: cosa ci dicono, prima facie, gli scritti celatiani sull'arte, sulla pittura? A voler rimanere a un livello puramente descrittivo, appare evidente l'attenzione dell'autore per gli "artisti-filosofi” (Brueghel ${ }^{3}$, Morandi, Giacometti), così come la preferenza per l'arte orientale (visibilissima fin dai saggi su Céline, e poi in quelli tardi su Michaux o Ariosto). Sono dati, del resto, che lasciano emergere una precisa disposizione riguardo al mondo della figuratività e delineano, anche e soprattutto, uno statuto "auspicabile" per la narrazione e il letterario. Nella riflessione di Celati, di fatto, l'arte che non sia afflitta dalla sindrome, tutta occidentale, della mimesi e del compiuto, produce e si allinea a una logica nuova, che dal punto di vista operativo è logica eidetica della congerie, dell'enciclopedico, del discontinuo; ed è invece, dal punto di vista dei suoi fondamenti, logica posizionale, logica del limite e dell'empiricosensorio.

Non stupisce, allora, che i riferimenti pittorici di Celati siano caratterizzati innanzitutto dall'ordinamento ritmico, più che prospettico, delle figure, $o$ ancora da un livello di astrattezza che vale per l'intrinseca "motricità" del gesto che questi stessi tratti disegnati sottendono, come in un certo espressionismo astratto americano. Allora, è vero che l'immagine e la figurazione dischiudono per Celati le possibilità di un nuovo intelletto, di una nuova modalità conoscitiva che trapassa qui, naturalmente, - e anche in questo Celati è erede di Calvino - in posizione etica, modalità di stare al mondo. Il discorso sull'immagine sembra in effetti tradursi in una questione d'ordine più generale e centrale in tutto il percorso intellettuale di Celati: la ricerca di un nuovo modo di approcciarsi al reale che non porti in sé la forma violenta delle gnoseologie razionalistiche, né la banalità dei codici preordinati di significazione. L'auspicio sotteso a tutto questo è allora che la scrittura stessa, come accennato in uno scritto su Wenders (Celati 2008b: 123), torni a essere pura "traccia" grafica: scarabocchio di per sé significante, che porti nella sua pochezza il segno dell'accettazione dell'esterno e dei fenomeni che ci abitano intorno.

Per quanto complesso, nella vastità, articolazione, dispersione di questo corpus, il nostro obiettivo è qui di leggere e incrociare gli scritti e appunti che Celati dedica ad artisti e pittori, tentando di definire i nuclei sostanziali di quello

appaiono tra virgolette basse uncinate; lì dove possibile, è indicata la datazione certa o plausibile tra parentesi quadre.

3 Si adotterà questo tipo di grafia per la trascrizione del nome dell'artista, salvo i casi in cui il nome compaia in citazione - e sarà allora privilegiata la forma scelta nel documento appunto citato. 
che appare, a tutti gli effetti, come un vero e proprio "pensiero delle immagini": ne abbiamo anticipato, per chiarezza e linearità, già i principali snodi, ma vorremmo ora illuminarne anche le suggestioni teoriche, le matrici generative, le consonanze rispetto al percorso evolutivo del Celati narratore e saggista.

Perché d'altronde l'immagine rientra, e rientra appunto su un piano di primordiale e persino ingenua vicinanza alla scrittura, fin nei primissimi progetti giovanili, del Celati redattore di Ali Babà, se è vero che a Oreste del Buono, in una lettera del '70, scriveva, a proposito della futura, incompiuta rivista: «Non so spiegarle bene lo spazio dell'illustrazione, ma sento che è decisivo» (Celati 1998b: 131). Ma il decennio dei Settanta, storicizzati ormai come annate del Celati carnevalesco e manicomiale, è il tempo in cui già emerge un rapporto complesso tra immagine e scrittura, che non si limiti a una funzione cioè meramente didascalica, ma sia già invece di un tipo particolare: una prossimità che apre al pensiero e all'elaborazione di una nuova teoria dell'espressione.

Non stupisce, allora, veder tornare con ripetuta insistenza nei primi scritti riferimenti all'art brut, al surrealismo e poi alla pop art: presenze pittoriche immediatamente comunicanti con la ricerca che in quegli anni Celati conduce sul linguaggio, a partire da una ferma critica al razionalismo cartesiano e calviniano. Comprensibile, allora, se si pensa a Comiche e al suo mondo verbo-delirante, il riferimento che nel saggio sul «Bazar archeologico» (Celati 2001d: 197-227) affiorerà a Adolf Wölfli e Dubuffet; e comprensibili, se si pensa alla ricezione della lezione benjaminiana, i riferimenti multipli a Lichtenstein, Warhol, Rauschenberg e Duchamp: tutte presenze insistenti in questo primissimo Celati, che significano già un'avvenuta presa di coscienza di quella che, sinteticamente, è stata qui definita logica del discontinuo.

Siamo a un cambio di prospettiva, quando Celati postilla L'oggetto surrealista di Gabellone (1977), già anticipato nei suoi clins d'œil alla tradizione pop e avanguardista da un saggio come «Il racconto di superficie» (Celati 1998c: 176193) e da alcune tracce residue al progetto Alì Babà: in tutti questi testi, l'arte contemporanea è, per Celati, denuncia degli infingimenti residui all'espressione, e insieme via e strumento d'affermazione d'un nuovo sguardo, "uno sguardo che [...] impedisce in sostanza di includere l'oggetto o il frammento in un ordine generalizzato» (Celati 1977: 153). È il passaggio, in breve, alla logica archeologica, che prelude all'instaurarsi non solo di nuove modalità espressive - il ready-made, l'arte Merz alla Kurt Schwitters ${ }^{4}$ - ma soprattutto, si diceva, di un nuovo modello conoscitivo; modello che, con Benjamin, superi ogni volontà sintetica, ogni ermeneutica preconcetta, per darsi piuttosto nei modi della Darstellung, dell'esposizione o illuminazione.

4 Schwitters è un altro autore che affiora spesso nelle riflessioni di Celati; ne parla ad esempio a proposito di Delfini: "Questo è ormai l'unico sfondo non pubblicitario dello scrivere. Delfini ne aveva fatto un suo campo ideativo, e in certi suoi quaderni c'è montaggio di pezzi sparsi che ricorda i collage di Schwitters» (Celati 2008a: 28). 
Nella rivalutazione della pop art e degli artisti duchampiani, si aggregano in Celati una serie di motivi concettuali che rimarranno vivissimi e presenti anche negli anni successivi. Scrive Celati nel Bazar, citando Oldenburg e George Brecht: «Da Baudelaire a Rimbaud a Breton a Duchamp alla Pop art, [si ha] la fine della poetica dell'occasione, del caso privilegiato [...] perché non v'è luogo o oggetto che non sia occasione: ognuno è simile e discontinuo, egualmente importante ma diverso rispetto all'altro» (Celati 2001d: 220). Non è solo la ripetuta insistenza sull'oggetto comune e anti-estetico, sul frammento e sul detrito, che pure richiama alla mente il Celati della "qualsiasità" neorealista; quanto il movimento anti-sintetico del pensiero che Celati invoca e ritrova in questi artisti, e riscoprirà poi in altri. Tutto punta a un appianamento delle gerarchie significanti, per la riscoperta, dirà poi lo stesso autore, dell'«ovvietà generale che ci accoglie nella vita quotidiana» (Celati 1998d: 318). Con ciò si ha, in breve, la valorizzazione di un metodo di indagine e riconoscimento del mondo che punti ad affrancarsi dalle logiche monovalenti, e ad accogliere invece un'istanza di "parificazione" e affratellamento dei diversi.

È la stessa logica che Celati sostiene in una lettera a Guido Neri e Calvino già nel 1969, quando parla delle «anatomie rinascimentali», delle «summe», delle «forme enciclopediche» (Celati 1998a: 109) come degli unici modelli di linguaggio in grado di sfidare il demone aristotelico della tassonomia: piuttosto che alla gerarchia, all'ordine precostituito e verticistico di valori, questi modelli puntano a costituirsi come «magazzini delle rimanenze» (Celati 2004: 185) - espressione con cui non a caso, anni più tardi, Celati chiamerà i paesaggi ritratti nelle fotografie di Ghirri. Il bazar figurativo degli artisti moderni - «da Schwitters a Cornell a Clarence Schmidt» (Celati 2008d: VIII) - è allora la via attraverso cui costruire la possibilità di uno sguardo nuovo, sfuggendo alle logiche preordinate, all'obiettività dei linguaggi, e abbracciando piuttosto i fenomeni nella loro complessità e vastità eteroclita.

A ben vedere, lo stesso tipo di polemica già emergeva nel saggio sul «Doppio parodico» (Celati 2001b: 111-163): qui, al principio del punto di vista unico viene opposto il colorito e bachtiniano mondo alla rovescia delle stampe fiamminghe, e soprattutto di Brueghel, come cantore di un sistema culturale e conoscitivo radicalmente opposto a quello, appunto monovalente, della tradizione urbana. Brueghel è artista ritornante nella parabola estetica e nella storia della ricezione di Celati. Non è un caso che buona parte della critica, a partire dall'autorevole precedente di Ghirri, abbia affiancato la narrativa celatiana ai quadri del pittore fiammingo. Aveva scritto Ghirri, all'uscita di Narratori delle pianure: «Mi vengono in mente, a proposito di questi racconti, soprattutto alcuni quadri di Bruegel, brulicanti di personaggi che si muovono in ogni direzione nello spazio che li contiene [...]» (Ghirri 2008a: 176)5.

5 Più recentemente, il paragone è stato ripreso da Patrick Barron e da Nunzia Palmieri a proposito dei Pascolanti. «Nella città di provincia si muovono, come in una visione di Bruegel gremita di personaggi, i tipi umani destinati a formare il grande quadro dei 
Quel che interessa, d'altronde, è questo: che, dagli anni Settanta fino agli ultimi scritti del 2000, l'analogia pittorica serva a Celati per puntualizzare una sua propria necessaria idea di scrittura: scrittura che resista ai facili smerci e si ponga, una volta di più, come "accumulo di roba sparsa". Scrive Celati a proposito di Tommaso Garzoni e della sua Piazza Universale: «È un panorama di parole, di figure, di maschere, di attività, di abitudini, di vizi, di aneddoti sparsi, che dà un'impressione simile a quella dei quadri di Brueghel» (Celati 2016c: 109). Di contro al pensiero monolitico, ai costrutti significativi, di contro soprattutto alle scritture letterarie e figurative che adottino la logica del punto di vista unico, Celati pone la riscoperta della Wunderkammer, del collage, del montaggio di reliquie, dell'accumulazione di resti, logica fieramente antiprospettica e anti-realistica, che trova il suo bersaglio polemico appunto in quella «sindrome», in quel «calvario» (sono tutti termini autoriali) che sembra affliggere, a leggere il saggio su «Beckett e le sorti dell'espressione», tutta l'arte dell'Occidente, dal Rinascimento in avanti.

Con Brueghel rivive in Celati una passione diremmo anti-rinascimentale, e invece quattrocentista: gotica, mistica, curiosa e pragmatica. Passione che si alimenta in gran parte del rifiuto del prospettivismo come criterio di fruizione e organizzazione del reale. Di Brueghel, Celati ammirava già negli anni Settanta lo «spazio senza un unico punto di fuga prospettico, che corrisponde ad una mancanza di agnizione univoca» (Celati 2001b: 158-159); e questo perché la polemica contro la totalizzazione dei significati rimane un punto centrale del suo pensiero, e si applicherà tanto a contesti figurativi, come in questo caso, che scrittòri, rifiutando quei «disegni costruttivi» che, ad esempio, nei «romanzi ben fatti», menino direttamente il lettore al «punto prospettico finale» (Celati 2016e: 243).

La sgranatura orizzontale, il pullulare dei dettagli, la superiorità del frammento sull'ordine unico, sono tutti elementi che Celati rimedita anche e soprattutto a partire dal visuale: dall'arte avanguardista, si è visto, non meno che dalla "pittura antica», definizione che ritorna con insistenza nei saggi degli anni Ottanta e Novanta, successivi all'incontro con Ghirri: nella prefazione al Profilo delle nuvole, e negli scritti su Tozzi, Stendhal, Delfini. Sono testi densi, che dischiudono una meditazione sul visivo difficilmente riportabile a cifra unica, ma a cui si può sempre tentare di rivolgersi almeno per frammenti. Nella prefazione a Con gli occhi chiusi, Celati scrive che «in Tozzi lo spazio si apre in ogni direzione e diventa spazio diffuso, come in una certa pittura antica, ad esempio Sassetta o Brueghel. E come nei quadri di quei pittori, nel nostro libro c'è a ogni pagina un pullulare di cose che parlano agli occhi, al di là dell'azione rappresentata - un migrare dell'attenzione da tutte le parti, un racconto che tende a spostarsi verso l'orizzonte» (Celati 2017: XV).

E solo uno tra i tanti spunti che vengono da un saggio interamente imbevuto, d'altronde, di riferimenti al visivo, e in cui tralucono evidenti i riferimenti alla

costumi degli italiani. È una piazza universale, come quella che si trova nel dizionario dei mestieri e delle professioni di Tomaso Garzoni [...]»(Palmieri 2012: 45). 
fenomenologia; ma al di là degli addentellati filosofici, al di là del raffinamento e della purificazione della scrittura saggistica celatiana, i riferimenti concettuali restano in fondo immutati rispetto al decennio precedente. Ritroviamo la stessa logica dell'appianamento, dell'apertura, dello «spazio gremito di cose», di "parvenze», dove tutto è predisposto in modo da sospendere il giudizio, o da evitare la caduta a picco verso la significazione unica. Il Tozzi di Celati è come Brueghel e Sassetta, artisti che arrivano tra queste pagine anche attraverso Ghirri, e che della fotografia di Ghirri costituiscono giustamente, come notava Quintavalle, le matrici pittoriche e strutturali.

Del saggio su Tozzi, d'altronde, è possibile individuare un precedente per così dire "esperienziale", puramente empirico, se si vada a guardare, ancora una volta, ai materiali d'archivio: il richiamo è qui a un'agendina del 1993, su cui Celati appunta osservazioni varie in seguito a una visita compiuta alla National Gallery di Londra. Davvero annotazioni brevi, che spesso ricadono nell'ekphrasis del quadro di volta in volta guardato, ma che immediatamente rinviano all'importanza davvero generativa dell'immagine, della pittura, per il pensiero della scrittura celatiano. Così, guardando Pisanello, Celati va con la mente a Boiardo, mentre "La battaglia di San Romano [di Paolo Uccello] evoca più Ariosto». A Tozzi, poi, Celati pensa rimirando la «serie del Sassetta sulla vite di San Francesco» (Celati 10/106: 3-5) ${ }^{6}$, quel Sassetta che Longhi opponeva, nella sua aria di "affinata spiritualità», nel suo ricorso a figurelle "slanciate ed estatiche» (Longhi 2013: 59ss), alla facoltà struttiva e all'implacabile concezione monumentale di un Giotto: e non è un caso, allora, che Celati, fermo avversatore di questa stessa "monumentalità" d'arte e di pensiero, si avvicini a un artista "florealista", che organizza spazi e paesaggi di modo da conferire alle scene un'aria di «favolosa irrealtà, [...] aerea vertigine, vibrante spaziosità»(Carli 1957: 70). Ma soprattutto un artista, citando Brandi, che rifugge alla disposizione geometrica delle figure, e in cui invece «tutto quello che appartiene apparentemente alla intelaiatura prospettica è risolto nella struttura ritmica» (Brandi citato in Carli 1957: 24), in puri valori di ritmo e linea: modi paradigmatici anche del narrare di Celati, a ben vedere, oltre che impliciti rimandi a quella teoria anti-prospettica che affiora a più riprese nelle sue meditazioni sull'immagine. Perché, nel nostro autore, la logica che sottende il visivo varrà anche come statuto auspicabile per la scrittura letteraria: è un "pensare per immagini” che si pone agli antipodi di ogni logica teorica, causale e discorsiva, e segue invece, come si è detto, una logica posizionale, accumulativa; serie di quadretti scenici che non convergono in un'ipotesi unica, ma si dispongono davanti all'occhio come «una forma d'ordine, una forma ritmica» (Celati 2008b: 187), scriverà l'autore sempre nel prezioso saggetto su Wenders.

Celati pensa a Tozzi tramite Sassetta perché lì, in quel ciclo pittorico, «l'anima mundi», «l'esser piantati nel mondo, come un albero o un sacco [...] si

6 La paginazione si intende a partire dall'inizio del brano manoscritto dedicato al «Viaggio alla National Gallery». 
fa racconto»; e perché, in Sassetta e in Tozzi, lo spazio «non [è] ancora disposto ad arte con un punto o un momento focale che attira gli occhi più degli altri» (Celati 10/106: 12-15). È il regno dell'eidetismo, appunta Celati: parola chiave che già si ritrovava addirittura nei saggi sulle Città invisibili di Calvino ${ }^{7}$, su Beckett degli anni Settanta ${ }^{8}$, su Garzoni e su Perec, e in cui in qualche modo convergono riflessioni sparse ma continue, condotte sul bordo tra immagine e parola. È l'idea di una forma espressiva che non si orienti verso codici e metasaperi positivi, ma si apra invece alla meraviglia del "mostrare", alla sparpagliata discontinuità del fenomeno.

Piero Camporesi, nel parlare di paesaggi e pittura, ricordava la vocazione universalistica che animava Leonardo e tutto il «matematico Quattrocento fiorentino»: vocazione nutrita da un naturalismo scientifico sensibile alla fenomenologia della natura, e che in qualche modo sembra, per vie traverse, giungere fino a Celati e alla sua volontà di «amare egualmente tutte le cose che si contengono nella pittura» (Leonardo Da Vinci citato da Camporesi 2016: 23-24) o nella scrittura. Ma siamo con Celati al totale allontanamento da presupposti di possesso o esaurimento descrittivo del mondo: la conoscibilità del reale è ormai modernamente accettata come parziale, e la sua rappresentabilità, dunque, dovrà emergere non come microscopica indagine del cosmo, ma invece solo come «traccia», traccia «afferrabile solo come effetto dell'apparire di qualcosa, il segno sulla tela o la frase sulla pagina dove prima non c'era nulla» (Celati 1/18: 13). Sono parole tratte dagli appunti preparatori per quello che diventerà il saggio «Beckett, le sorti dell'espressione» (Celati 1986): saggio denso e complesso, che postilla le traduzioni dell'autore ai Three Dialogues di Beckett e ad altri suoi due pamphlet sulla "pittura moderna». Un saggio, tra l'altro, che ha alle spalle non solo Merleau-Ponty, ma anche i filosofi anglosassoni del linguaggio, e Wittgenstein poi tra tutti. Qui trova piena formulazione quanto rimaneva allo stato di accenno negli scritti su Tozzi e Sassetta: e cioè la polemica contro il «calcolo prospettico» che vincola la visione, "come del resto la letteratura», a un'azione e un progetto costruttivo9. Ma soprattutto è qui che si addensa, attraverso Beckett che guarda all'olandese Bram Van Velde, - ma non è l'unico riferimento, perché Celati cita anche Kandisky, Mirò, Rouault, Baj, Bacon, Vedova e Gnoli - una meditazione

7 «L'eidetismo è la qualità specifica della superficie, perché infittendo i dettagli da percorrere abolisce ogni possibilità di percezione globale» (Celati 1998c: 189).

8 «Una specie di eidetismo in quanto mantiene la registrazione di particolari insignificanti, ma manca di un pattern di memorizzazione del tutto» (Celati 2001c: 175-176).

9 «L'arte occidentale, a partire dal rinascimento italiano, non ha mai smesso di essere concepita come un progetto per costruire la visione, sia grazie al calcolo prospettico, sia grazie al discorso critico che diventa il piano portante d'una costruzione culturale. In ogni caso la pittura, come del resto la letteratura, non ha mai smesso di essere proposta come rinnovamento d'una costruzione per guidare l'occhio e il pensiero figurale. Per installarli in un campo codificato, per ridurre tutto lo spazio a linee di fuga che servono a determinare in modo permanente gli oggetti della visione» (Celati 1/20-21: 7). 
centrale sull'“espressione” come forma di linguaggio in sé limitata. La pittura del moderno dimostra quanto ipocritamente infondate fossero le «teorie della rappresentazione tradizionali o realistiche», quelle teorie cioè che, dominate dall'ipoteca mimetica, dall'illusiva corrispondenza tra nomi e cose, dal falso «rapporto di buona adeguazione tra una forma espressiva e ciò a cui questa forma si apre» (Celati 1/18: 22), hanno per anni condannato l'arte occidentale al calvario, alla sindrome e al dilemma dell'espressione: a formulare cioè il rapporto tra espressione e cosa espressa in termini via via analogici, teologici, scientifici, e sempre declinandolo, nella sua problematicità, come negativo; senza capire, scrive Celati in questi primissimi appunti dattiloscritti, «che in realtà non c'è niente da esprimere. Perché l'espressiane [sic] non è qualcosa che abbia un contento $[\mathrm{sic}]$, un dare e un avere, ma è piuttosto una tensione che cozza contro un impedimento ed un limite che deve accettare» (Celati 1/18: 20-21).

A partire da Beckett e dai quadri dei fratelli Van Velde, Celati giunge a elaborare profondamente la questione del fallimento e dell'indigenza dell'espressione, espressione che accetta «la propria miseria, la propria pochezza, senza più sognare l'inesprimibile» (Celati 1/18: 21). Ed è una pochezza del tutto positiva, che si pone in polemica contro la catalogazione nominalistica del mondo, e si schiera a favore dell'accettazione percettiva delle cose esterne, così come del «limite d'espressione» dei nostri «artefatti di parole o di immagini»: "Se tutto ciò che spunta dalla penna o dal pennello cade entro i propri limiti, ossia comunque "viene come viene", è questo apparire di qualcosa che conta come lavoro o quadro o scrittura, e non il delirio d'una pienezza che dovrebbe esprimere» (Celati 1/18: 43). Con ciò, l'arte astratta moderna non punta, nel Beckett riletto da Celati, nella direzione di un'autogiustificazione per via simbolica o trascendentalista: al contrario, mostra la via "nuova" di un'espressione libera da ipoteche preconcette e pretese categorizzanti, tutta giocata nei limiti dell' «immanenza scopica (la visione che si volge al qualsiasi dell'immanenza, senza giudizi di valore precostituiti)»(Celati 2002: 196-196) o di quella che viene definita una «trascendenza profana» (Celati 2006: XXIV). E ci sembra oltretutto indicativo che in queste carte, più volte rimaneggiate dall'autore, risulti centrale l'idea della positività del limite, dell'indigenza, della pochezza: le stesse parole che torneranno, più puntuali e organizzate, a definire l'idea di scrittura letteraria in un testo manifesto qual è la prefazione al Bartleby di Melville - dove si dice che la potenza della scrittura «non sta in questa o quella cosa da dire, bensì nel poco o niente da dire, in una condizione in cui si annulla il dovere di scrivere» (Celati 2006: XXVI).

È questo forse uno degli altri "pioli" su cui si regge per intero la meditazione di Celati sul visivo, riportabile a quella che è stata qui definita come logica del limite, e che continuamente delinea e suggerisce uno spartiacque tra nomiintesi come universali filosofici - e immagini, che hanno in Celati sempre una dimensione contingente e pragmatica. Lo si dice in maniera esplicita, citando Morandi, Cézanne, Giacometti, in un altro quadernino d'archivio degli anni Novanta: «l'esperienza dell'immagine» ha attinenza col fallimento, con «la 
loro [di questi pittori] esperienza di un'impossibilità in cui va a parare tutto quello che percepiamo» (Celati 2/35: 3), e con la loro fuga da ogni pretesa autoconsolatoria di allineare linguaggio e mondo.

Una simile postura, oltre a delineare un'intrinseca contrapposizione tra logica discorsivo-concettuale e logica dell'immagine - come logica invece del puro apparire, logica spaziale, pragmatica e a-significante -, riemerge in tutto l'insieme delle altre riflessioni celatiane sull'arte: è ben presente, ad esempio, nell'insieme di appunti dattiloscritti dedicati al pittore normanno Jacques Deschamps, di cui l'autore visita una mostra a Caen tra il 1987 e il 1988 (subito dopo, dunque, aver meditato i pamphlet beckettiani sulla pittura moderna). Commentando l'arte cosiddetta "astratta" di Deschamps, Celati torna a parlare dell'aspetto tattile, sensitivo, artigianale di queste tracce pittoriche: «Ciò che fa pittura, per lui, è una superficie guardabile. È la superficie della tela, sottratta alle sovrastrutture simboliche, comprese quelle della figurazione»; e ancora «[nei] suoi quadri, [...] l'aspetto tattile e il gusto per la trama delle cose rendono tutto quello che vedo concreto, guardabile senza bisogno di concetti, più che mai nell'ordine del sensibile» (Celati 8/17: 1).

E quest'idea di un "fare" pittorico che si sottragga agli imperativi della significazione, che dischiuda l'orizzonte di un pensiero a-concettuale, e che possa comprendersi soltanto a partire da un'istanza percettivo-corporale, si ripresenta e converge in Celati nella rivalorizzazione costante dell'arte orientale, del puro motivo decorativo, fino al limite estremo costituito dallo scarabocchio. Sono dettagli rilevabili, come si diceva, fin dai saggi su Céline, quando la féérie stilizzata dei suoi romanzi veniva espressamente associata all' "arte orientale», alle "maschere [...] ghignanti [...] del teatro no» giapponese, e poi di nuovo a «Brueghel e Bosch» (Celati 1996: XII-XXV), realizzando a pieno, per altro, quel nesso che già Jurgis Baltrušaitis aveva illustrato tra gotico europeo e tradizioni figurative asiatiche. E poi negli interventi dei tardi anni Novanta e dei Duemila, quando i principi posizionali e panoramici del novellare antico e dell'epica ariostesca vengono riportati, con un'altra piroetta analogica, all'immagine dell'arabesco, dell'intrico di linee simile a quello dei «versetti coranici» (Celati 2016e: 264), dei «manoscritti persiani» o dei «tappeti d'Oriente o [...] alessandrini» (Celati 2016b: 80ss): forma espressiva, dice Celati, svestita da ogni velleità di rappresentazione, e mossa unicamente da linee tensive, da criteri non logici o causali, ma puramente spaziali e relazionali. Qui sembra addirittura riaffiorare il Celati lettore di Deleuze e dei linguisti; e torna anche, arricchito di nuove suggestioni, forte della complessa riflessione condotta su Bram Van Velde, il Celati estimatore delle grandi «summe» e delle Wunderkammer. Logica del limite e logica del discontinuo si uniscono qui, dunque, nella riqualificazione delle scritture - verbali, non meno che grafiche - che si pongono come atlanti brulicanti di punti e linee. E il parallelo con la pittura orientale diventa flagrante, una volta di più, nelle tarde riflessioni condotte su Michaux, non a caso poetapittore, i cui «disegni paiono formicai, masse di figurine ridotte all'essenziale», 
segni, tracce che si perdono, «come nella pittura cinese», in una «lontananza evanescente» (Celati, Talon 2005: 228ss).

A quest'altezza, la convergenza tra immagine e parola arriva a un altro punto di tensione, per risolversi quasi compiutamente nell'idea dello scarabocchio, della traccia inchiostrata che sul quadro, o sulla pagina, realizza una perfetta corrispondenza tra intenzione, messaggio e forma ornamentale. Un tipo di coincidenza che Celati invocava già addirittura nel saggio sul «Racconto di superficie», e che tornerà poi a visitarlo negli anni. Quel che importa, insomma, è che dietro la traccia ci sia il gesto, si avvertano la mano e il suo umore: idea questa, su cui si era già innestato il pensiero di Celati a partire dal saggio su «Beckett, l'interpolazione e il gag», ma che non sembra affatto diminuire negli anni, e anzi sembra arricchirsi di nuovi attributi, proprio grazie all'insistenza del paragone figurale. Allora, ad esempio, i segni di Michaux valgono perché procedono da un puro "stanziamento sulla carta», sono puri «gesti», «movimenti» sulla pagina (Celati, Talon 2005: 228): e questo, come nella pittura concreta di Deschamps, e nel disegno a trame orientale, non fa che esplicitare per Celati l'unico, salutare, modo d'essere dell'espressione. Dove la scrittura diventa cioè puro grafismo, traccia che trattiene in sé la spontaneità del gesto, «segno decorativo, arzigogolo, segno runico, segno fatato, liquidazione a sorpresa di tutta la coscienza raziocinante» (Celati 2016d: 144). Parole, queste, tratte alla prefazione al Mastr'Impicca di Vittorio Imbriani, scritta nel '98: un testo, forse, che più di altri rivela la costante preoccupazione di delineare per la scrittura uno statuto a un tempo figurativo e diminutivo.

Ed è così che l'immagine, da sostegno secondario a una presupposta univoca priorità di significati, diventa per Celati essa stessa significato, nuova forma di intelletto: è in questo spazio, scrive l'autore commentando Nel corso del tempo, che si potrà positivamente regredire al "pensiero infantile per immagini», a quel tempo cioè in cui le lettere dell'alfabeto vengono immaginosamente recepite come figure fantastiche, prima dell'ottundimento provocato nell'adulto dai «sistemi di parole scritte» (Celati 2008b: 123). Sembra quasi veder riecheggiare qui alcune costanti del pensiero antropologico, sulla scrittura come "addomesticamento della cognizione" e sul valore performativo, mimico e causativo della parola-gesto.

$\mathrm{Ma}$ in Celati è attiva, come si diceva, anche una costante ricerca sulle pratiche conoscitive: per cui la meditazione sulla pittura, le riflessioni sull'immagine, si inquadrano in una precisa forma di ricerca sulle nostre modalità di conoscere e di relazionarci al reale. Non a caso Celati cita Giacometti - «Io disegno per capire cosa vedo» (Celati 2008c: 128) - parlando di Ghirri, e non a caso Ghirri citava Giordano Bruno a proposito dell'essenza delle immagini, definite dal gran nolano come «enigmi che si risolvono col cuore» (Ghirri 2008b: 131): in entrambi i casi si fa riferimento all'insito potere orientativo della visione, e insieme all'implicito nesso che questa forma di conoscenza intrattiene con il campo delle passioni, delle simpatie e delle proiezioni empatiche. 
Siamo, con l'immagine, e con la scrittura che auspica a farsi essa stessa immagine, alla dismissione del sapere generalista, e all'apertura invece di una nuova logica: pratica, empirica, sensoriale. Anche qui il gioco è nel rovesciamento dei presupposti cristiano-cartesiani di una supposta primazia dell'interiorità a scapito di ciò che è esterno e per così dire "superficiale": una riscoperta, di contro alle filosofie essenzialistiche, dell'immagine come spunto e apertura a una sensitività immaginale che cortocircuita, una volta e per sempre, la polarizzazione tra oggetto e soggetto, tra percepito e percipiente, puntando invece in primo luogo alla possibilità di ricostituire il nesso tra conoscere e esperire. È certo un portato della lezione merleau-pontiana, si diceva, ma che trova anche significativi, innegabili punti di contatto con le più recenti acquisizioni della neo-fenomenologia ${ }^{10}$, e con le riflessioni condotte da ultimo - a partire da una profonda rilettura dell'aristotelismo averroista - da uno studioso come Emanuele Coccia ${ }^{11}$ : in breve, ci sembra che Celati tenda sempre, peculiarmente, a ritrovare quel punto di contatto, quella saldatura tra vita e espressione, capace di rendere la scrittura un luogo di adesione al reale; adesione al reale, tuttavia, non in un senso naturalistico o descrittivo, ma appunto, piuttosto, come forma intellettiva e come manière d'être, nuova modalità di stare al mondo. Per cui, appunto, l'immagine - sia essa pittura, apparizione, referente ottativo per la parola letteraria - si configura sempre in Celati come possibilità di evasione da una teoria "fissista" dell'esistere, come luogo di rifiuto di un'estetizzazione forzata e precostituita del mondo, e come portale di una modalità d'essere e di esperire il mondo stesso che sia radicalmente pre-teorica, e invece estesiologica ed emotivamente connotata.

\section{Bibliografia}

Baltrušaitis J., 1955, Le Moyen Âge fantastique: Antiquités et exotisme dans l'art gothique, Paris, Colin.

Barron P., 2007, «Gianni Celati's Poetic Prose: Physical, Marginal, Spatial», Italica, 2-3, p. 323-344.

Belpoliti M., [1996] 2006, L'occhio di Calvino, Torino, Einaudi.

Camporesi P., [1992] 2016, Le belle contrade, Milano, Il Saggiatore.

Carli E., 1957, Sassetta e il maestro dell'osservanza, Milano, Aldo Martello.

Celati G., 1977, «Prefazione», in Gabellone L., L'oggetto surrealista, Torino, Einaudi, p. 149-154.

10 Pensiamo soprattutto agli studi sul concetto di atmosfera, e a quella congiuntura con le riflessioni di Tonino Griffero che già Marina Spunta notava in suo contributo sul cinema celatiano, cfr. Spunta 2008.

11 Celati legge e conosce almeno la prima importante opera dell'autore, La trasparenza delle immagini (2005); ne fa cenno, quello stesso anno, a Massimo Rizzante, in occasione di un incontro tenutosi all'Università di Trento, e di cui si leggono le tracce nel saggio-intervista «Sulla fantasia» (Celati 2011: 72). 
Celati G., 1986, «Samuel Beckett, le sorti dell'espressione», In forma di parole, 1, p. 259-268.

Celati G., 1996, «Prefazione», in Céline L.F., Guignol's band I-II preceduti da Casse-pipe, Torino, Einaudi-Gallimard, p. IX-XXXIV.

Celati G., [1969] 1998a, "Gianni Celati a Guido Neri e Italo Calvino», Riga, 14, "Ali Babà". Progetto di una rivista. 1968-1972, a cura di M. Barenghi e M. Belpoliti, p. 107-110.

Celati G., [1970] 1998b, "Gianni Celati a Oreste Del Buono (2 ottobre 1970)», Riga, 14, "Ali Babà". Progetto di una rivista. 1968-1972, a cura di M. Barenghi e M. Belpoliti, p. 131-132.

Celati G., [1973] 1998c, «Il racconto di superficie», Riga, 14, "Ali Babà". Progetto di una rivista. 1968-1972, a cura di M. Barenghi e M. Belpoliti, p. 176-193.

Celati G., 1998d, «Il progetto “Ali Babà”, trent'anni dopo», Riga, 14, "Ali Babà”. Progettodiuna rivista. 1968-1972, a curadiM. BarenghieM.Belpoliti,p. 313-321.

Celati G., 2001a, Finzioni occidentali: Fabulazione, comicità e scrittura, Torino, Einaudi.

Celati G., [1975] 2001b, «Il tema del doppio parodico», in Celati 2001a, p. 111-163. Celati G., [1975] 2001c, «Su Beckett, l'interpolazione e il gag», in Celati 2001a, p. 165-194.

Celati G., [1986] 2001d, «Il bazar archeologico», in Celati 2001a, p. 195-227.

Celati G., 2002, «Nota su Flann O’Brien e il suo traduttore», in O'Brien F., L'ardua vita. Esegesi dello squallore, Varese, Giano, p. 191-196.

Celati G., [1989] 2004, "Commenti su un teatro naturale delle immagini», in Sironi M., Geografie del narrare, Reggio Emilia, Diabasis, p. 178-189.

Celati G., [1991] 2006, «Introduzione», in Melville H., Bartleby lo scrivano, Milano, Feltrinelli, p. VII-XXVI.

Celati G., 2008a, «Letteratura come accumulo di roba sparsa. Conversazione con Marco Belpoliti e Andrea Cortellessa», Riga, 28, Gianni Celati, a cura di M. Belpoliti e M. Sironi, p. 25-37.

Celati G., 2008b, "Quando ho visto "Nel corso del tempo"», Riga, 28, Gianni Celati, a cura di M. Belpoliti e M. Sironi, p. 122-125.

Celati G., 2008c, "Viaggio in Italia con 20 fotografi, 20 anni dopo», Riga, 28, Gianni Celati, a cura di M. Belpoliti e M. Sironi, p. 126-135.

Celati G., 2008d, "Antonio Delfini ad alta voce», in Delfini A., Autore ignoto presenta, Torino, Einaudi, p. V-XXXV.

Celati G., [2005] 2011, «Sulla fantasia», in Id., Conversazioni del vento volatore, Macerata, Quodlibet, p. 70-80.

Celati G., 2016a, Studi d'affezione per amici e altri, Macerata, Quodlibet.

Celati G., [2000] 2016b, «Angelica che fugge», in Celati 2016a, p. 49-103.

Celati G., [2001] 2016c, «La piazza universale di tutti i mestieri», in Celati 2016a, p. 105-121. 
Celati G., [1998] 2016d, «Imbriani, il favolare, l'ingenuità e lo scarabocchio», in Celati 2016a, p. 123-147.

Celati G., [1998] 2016e, «Discorso sull'aldilà della prosa», in Celati 2016a, p. 239273.

Celati G., [1994] 2017, «Prefazione», in Tozzi F., Con gli occhi chiusi, Milano, Feltrinelli, p. VII-XXIV

Celati G., s.d., «Samuel Beckett, le sorti dell'espressione», busta 1/18, Fondi d'archivio, Reggio Emilia, Biblioteca Panizzi, 52 carte sciolte dattiloscritte.

Celati G., s.d., «Introduzione», busta 1/20-21, Fondi d'archivio, Reggio Emilia, Biblioteca Panizzi, 12 carte dattiloscritte.

Celati G., [1987 ca], «Scritti sul pittore Jacques Deschamps», busta 8/71, Fondi d'archivio, Reggio Emilia, Biblioteca Panizzi, 13 carte sciolte dattiloscritte.

Celati G., [1992-1993 ca], "Testo mutilo sulle immagini, sui nomi che si danno alle cose e sull'esperienza dell'immagine», busta 2/35, Fondi d'archivio, Reggio Emilia, Biblioteca Panizzi, 3 carte sciolte dattiloscritte.

Celati G., [1993], «Riflessioni sui modi di rappresentazione, sullo scrivere, sul dipinto San Gerolamo di Antonello da Messina, conservato alla National Gallery», busta 10/106, Fondi d'archivio, Reggio Emilia, Biblioteca Panizzi, agendina con copertina rigida, inchiostro manoscritto.

Celati G. e Talon J., [2005] «Presentazione di Henri Michaux», in Michaux H., Altrove. Viaggio in Gran Garabagna. Nel paese della Magia. Qui Poddema, Macerata, Quodlibet, p. 215-245.

Coccia E., 2005, La trasparenza delle immagini: Averroè e l'averroismo, Milano, Mondadori.

Cortellessa A., 2005, «Frammenti di un discorso sul comico. "Archeologia di un'archeologia” per Gianni Celati: 1965-78», in Cirillo S., Il comico nella letteratura italiana: teorie e poetiche, Roma, Donzelli, p. 543-574.

Ghirri L., [1985] 2008a, «Una carezza al mondo», Riga, 28, Gianni Celati, a cura di M. Belpoliti e M. Sironi, p. 176.

Ghirri L., [1989] 2008b, Il paesaggio impossibile, in Id., Bello qui, non è vero?, Roma, Contrasto, p. 131.

Griffero T., [2010] 2017, Atmosferologia. Estetica degli spazi emozionali, MilanoUdine, Mimesis.

Longhi R., [1914] 2013, Breve ma verifica storia della pittura italiana, Milano, Abscondita.

Mussini M., 2001, «Luigi Ghirri. Attraverso la fotografia», in Luigi Ghirri, a cura di Massimo Mussini, Milano, Federico Motta, p. 9-49.

Palmieri N., 2012, "Note sui "Pascolanti” di Gianni Celati», Oblio - Osservatorio Bibliografico della Letteratura Italiana Otto-novecentesca, 5, p. 44-48.

Quintavalle A., 1992, "Lo sguardo di Ghirri. Per una comédie humaine sulle rive di un antico fiume», in Ghirri P. e Taramelli E., Luigi Ghirri. Vista con camera. 200 fotografie in Emilia Romagna, Milano, Motta, p. 199-203. 
Spunta M., 2008, "Verso la foce" con Gianni Celati: tra "antropologia di popolazioni invisibili" e "estetica del reincanto"”, in Bonsaver G., McLaughlin M. e Pellegrini F., Sinergie narrative: Cinema e letteratura nell'Italia contemporanea, Firenze, Franco Cesati, p. 153-167. 